\title{
Electronically Tunable Perfect Absorption in Graphene
}

Seyoon Kim, Min Seok Jang, Victor W. Brar, Kelly W Mauser, Laura Kim, and Harry A Atwater

Nano Lett., Just Accepted Manuscript • DOI: 10.1021/acs.nanolett.7b04393 • Publication Date (Web): 10 Jan 2018

Downloaded from http://pubs.acs.org on January 11, 2018

\section{Just Accepted}

"Just Accepted" manuscripts have been peer-reviewed and accepted for publication. They are posted online prior to technical editing, formatting for publication and author proofing. The American Chemical Society provides "Just Accepted" as a free service to the research community to expedite the dissemination of scientific material as soon as possible after acceptance. "Just Accepted" manuscripts appear in full in PDF format accompanied by an HTML abstract. "Just Accepted" manuscripts have been fully peer reviewed, but should not be considered the official version of record. They are accessible to all readers and citable by the Digital Object Identifier (DOI®). "Just Accepted" is an optional service offered to authors. Therefore, the "Just Accepted" Web site may not include all articles that will be published in the journal. After a manuscript is technically edited and formatted, it will be removed from the "Just Accepted" Web site and published as an ASAP article. Note that technical editing may introduce minor changes to the manuscript text and/or graphics which could affect content, and all legal disclaimers and ethical guidelines that apply to the journal pertain. ACS cannot be held responsible for errors or consequences arising from the use of information contained in these "Just Accepted" manuscripts. 


\title{
Electronically Tunable Perfect Absorption in Graphene
}

\author{
Seyoon Kim ${ }^{\dagger,+}$, Min Seok Jang ${ }^{\dagger,+,+}$, Victor W. Brar ${ }^{\dagger,,_{,},+}$, Kelly W. Mauser ${ }^{\dagger}$, Laura Kim ${ }^{\dagger}$, and \\ Harry A. Atwater ${ }^{\dagger, \S, *}$ \\ *haa@caltech.edu \\ ${ }^{+}$Equally contributed authors
}

† Thomas J. Watson Laboratory of Applied Physics, California Institute of Technology, Pasadena, CA 91125, United States

${ }^{\ddagger}$ School of Electrical Engineering, Korea Advanced Institute of Science and Technology, Daejeon 34141, Republic of Korea

${ }^{\S}$ Kavli Nanoscience Institute, California Institute of Technology, Pasadena, CA 91125, United States

"Department of Physics, University of Wisconsin-Madison, Madison, WI 53711, United States 


\begin{abstract}
The demand for dynamically tunable light modulation in flat optics applications has grown in recent years. Graphene nanostructures have been extensively studied as means of creating large effective index tunability, motivated by theoretical predictions of the potential for unity absorption in resonantly-excited graphene nanostructures. However, the poor radiative coupling to graphene plasmonic nanoresonators and low graphene carrier mobilities from imperfections in processed graphene samples have led to low modulation depths in experimental attempts at creating tunable absorption in graphene devices. Here we demonstrate electronically tunable perfect absorption in graphene, covering less than $10 \%$ of the surface area, by incorporating multi-scale nanophotonic structures composed of a low permittivity substrate and subwavelength noble metal plasmonic antennas to enhance the radiative coupling to deep subwavelength graphene nanoresonators. To design the structures, we devised a graphical method based on effective surface admittance, elucidating the origin of perfect absorption arising from critical coupling between radiation and graphene plasmonic modes. Experimental measurements reveal $96.9 \%$ absorption in the graphene plasmonic nanostructure at $1,389 \mathrm{~cm}^{-1}$, with an on/off modulation efficiency of $95.9 \%$ in reflection.
\end{abstract}

KEYWORDS: Graphene, plasmonics, perfect absorption, tunable resonance, mid-infrared, optical modulator 
Over the past decade, advanced fabrication technologies have enabled enormous progress in developing nanophotonic metasurfaces for tunable optical devices through gate-tunable indexchanging materials. Graphene has been the subject of intensive research as a promising candidate for tunable amplitude and phase modulation of $\mathrm{THz}$ and mid-infrared light ${ }^{1-6}$. In these frequencies ranges, the dielectric constant of graphene is dynamically tunable, via modulation of the carrier density under electrostatic gate control, which allows/forbids for the presence of plasmons in the graphene sheet. These plasmons display extreme light confinement, which allows the graphene sheet to be patterned at extremely small length-scales, while sill interacting dynamically with longer wavelength light ${ }^{7-22}$. In particular, absorption strength in graphene is one of the most important measure for control of photon harvesting, and theoretical schemes have tantalizingly predicted $100 \%$ absorption in resonantly excited graphene nanostructures ${ }^{15,16}$, indicating strong light-matter interactions in atomically thin layers.

Despite these exciting predictions and attractive features of tunable graphene nanostructures, a major obstacle for realizing perfect absorption in graphene has been the low carrier mobility in processed graphene samples due to PMMA residues ${ }^{23}$ and/or trapped impurities $^{24}$ as compared with the high carrier mobilities achievable in pristine or passivated and unpatterned graphene sheets sheets ${ }^{25,26}$, which have been assumed in theoretical works predicting unity absorption. In addition, edge defects in patterned graphene ${ }^{27-30}$ and large carrier densities ${ }^{31}$ required for exciting graphene plasmons also degrade effective graphene carrier mobilities. The low graphene carrier mobility gives rise to a high non-radiative damping rate, which tends to under-couple the resonant modes of graphene nanoresonator arrays ${ }^{32}$. Since large-area high performance functional graphene devices cannot rely on the high carrier mobility of exfoliated graphene flakes, achieving this performance level with graphene synthesized by chemical vapor deposition -with its attendant lower carrier mobility- is important.

One way to circumvent this issue is to increase radiative coupling to graphene nanoresonators, which is generally weak due to the inherent thinness of graphene as well as the large wavevector mismatch between graphene plasmons and free-space photons. To date, various methods, including large chemical doping ${ }^{7-9}$, carefully designed substrates ${ }^{14-16}$, and integrated noble metal plasmonic structures ${ }^{17-21}$, have been explored to increase coupling of radiation to graphene nanoresonators. In spite of these efforts, the realistic problems in processed graphene 
nanostructures have limited the maximum experimentally achievable absorption in graphene, and not even $50 \%$ absorption in graphene has been realized in the mid-infrared.

In this paper, we show the experimental demonstration of electronically tunable perfect absorption in graphene through graphene plasmonic nanostructures that exhibit dramatically higher resonant absorption, made possible by tailored nanophotonic designs that leverage the atomic thinness of graphene and the extreme confinement of graphene plasmons. First, we utilize lower permittivity substrates, which allow better wavevector matching between free-space photons and graphene plasmons, to improve radiative coupling to graphene plasmonic ribbons (GPRs). Second, we combine the GPRs with noble metal plasmonic metallic antennas as subwavelength-scale intermediaries $\left(\sim \lambda_{0} / 10, \lambda_{0}\right.$ : free-space wavelength) to further enhance radiative coupling to deep subwavelength-scale GPRs $\left(<\lambda_{0} / 70\right)$.

Unlike other perfect absorbers solely relying on noble metal plasmonic effects ${ }^{17,32}$, our structures create perfect absorption in the graphene itself by utilizing graphene plasmonic resonances, providing an ideal platform for tunable strong light-matter interactions. Of note, tunable perfect absorption is achieved with graphene nanoresonators covering less than $10 \%$ of the surface area, whereas unpatterned graphene sheets exhibit low single-pass absorption $(\sim 2.3 \%)$.

To design our plasmonic nanostructures, we devised a graphical method based on effective surface admittance. This graphical approach serves to elucidate the underlying physics of the perfect absorption in graphene and the role of other elements of our multi-scale structure, revealing that perfect absorption is achieved when the graphene plasmonic nanostructure is carefully tailored to induce critical coupling to free space, i.e., matching of the admittance of graphene resonators to free space. This theoretical model reveals that perfect absorption is no longer limited by low graphene carrier mobility. As an example, we present calculations predicting perfect absorption at mid-infrared wavelengths for a graphene hole mobility as low as $200 \mathrm{~cm}^{2} \mathrm{~V}^{-1} \mathrm{~s}^{-1}$ and at a moderate doping with the graphene Fermi level position less than $-0.6 \mathrm{eV}$, which are more realistic conditions than those theoretically proposed to realize perfect absorption. Finally, reflection measurements for prototype structures demonstrate that electronically tunable resonant absorption can be increased from $24.8 \%$ to $96.9 \%$ at $1,389 \mathrm{~cm}^{-1}$, with an on/off modulation efficiency of $95.9 \%$ in reflection. 
Figure 1 shows three designs for perfect absorption structures incorporating GPRs. All structures utilize a $\mathrm{SiO}_{2} / \mathrm{SiN}_{x}$ membrane with a back reflector as a base, which creates a "Salisbury screen" effect to enhance absorption in the GPRs ${ }^{15,16,33}$ (Supporting Information Part 1). The type A structure depicted in Figure 1a consists of periodically arrayed $100 \mathrm{~nm}$ gap/100 $\mathrm{nm}$ wide GPRs on the $\mathrm{SiO}_{2} 150 \mathrm{~nm} / \mathrm{SiN}_{x} 1 \mu \mathrm{m} / \mathrm{Au}$ substrate. The type $\mathrm{B}$ and $\mathrm{C}$ structures on the $\mathrm{SiO}_{2} 150 \mathrm{~nm} / \mathrm{SiN}_{x} 500 \mathrm{~nm} / \mathrm{Au}$ substrates in Figure 1b,c have GPRs located inside subwavelength metallic slits. In the type B structure, a $100 \mathrm{~nm}$ wide GPR is located in the center of a $200 \mathrm{~nm}$ wide metallic slit, while the metallic slit is $100 \mathrm{~nm}$ wide and the $50 \mathrm{~nm}$ wide GPR is off to one side in the type $\mathrm{C}$ structure. The widths of the $80 \mathrm{~nm}$ thick metallic strips making up the slits in the type $\mathrm{B}$ and $\mathrm{C}$ structures are $910 \mathrm{~nm}$ and $615 \mathrm{~nm}$, respectively. The total area for each structure is about $75 \mu \mathrm{m} \times 75 \mu \mathrm{m}$, and scanning electron microscope images are shown in Figure 1d-f for all structures.

The geometries of all structures were chosen in order to display maximum absorption at $1,356 \mathrm{~cm}^{-1}$ with graphene Fermi level below $-0.6 \mathrm{eV}$, similar to the conditions for enhanced absorption that we previously reported for a conventional GPR structure on a $\mathrm{SiN}_{x} / \mathrm{Au}$ substrate $^{16}$, which we refer to here as the type 0 structure. Electric field profiles with graphene plasmonic resonance in the type A-C structures are presented in Figure 1g-1. Enhanced electric fields around the GPRs in Figure 1g-i indicate strong coupling of radiation into graphene plasmon modes. Figure $1 \mathrm{j}-1$ shows a collective oscillation in the type A structure due to the interaction between adjacent GPRs, and equivalent collective oscillations in the type B and C structures created by image GPRs inside the metallic strips of Figure 1b,c. The details on the equivalent collective oscillations in the type B and C structures will be discussed below.

Varying the substrate and surface environment can yield tangible benefits towards the goal of achieving perfect absorption in electrostatically gated graphene plasmonic nanostructures with lower graphene carrier mobilities. To evaluate the improvement by multi-scale nanophotonic structures, we consider as a key metric the graphene carrier mobility required to achieve perfect absorption in each structure, and show that perfect absorption can be achieved with the lower graphene carrier mobilities typically seen in transferred and lithographically processed graphene. One of the fundamental problems that leads to low absorption in graphene plasmonic structures is the large wavevector mismatch between free-space photons and graphene plasmons $^{1-3}$. While such a property of graphene plasmons allows miniaturizing optical elements, 
it leads to inefficient radiative coupling to graphene nanoresonators. Because the dispersion relation of a transverse magnetic graphene plasmon is dependent on substrate permittivity ${ }^{34-36}$, graphene plasmons will have a smaller wavenumber on a lower permittivity substrate, thus reducing the wavevector mismatch between free-space photons and graphene plasmons, resulting in higher radiative coupling efficiency. In addition, the reduced wavevector mismatch via a low permittivity substrate make the fabricated structures more robust to fabrication imperfections which cause inhomogeneous broadening. The details on the effect of a low permittivity substrate are discussed in Supporting Information Part 3.

To exploit this property, $\mathrm{SiO}_{2}$ layers are incorporated in the type A-C structures beneath the GPRs, while the GPRs of the type 0 structure lie on a $\operatorname{SiN}_{x}$ layer possessing higher permittivity than $\mathrm{SiO}_{2}$. By incorporating the low permittivity layer, the type A structure exhibits perfect absorption for a graphene hole mobility $\left(\mu_{\mathrm{h}}\right)$ of $2,271 \mathrm{~cm}^{2} \mathrm{~V}^{-1} \mathrm{~s}^{-1}$, while $\mu_{\mathrm{h}}=3,174 \mathrm{~cm}^{2} \mathrm{~V}^{-1} \mathrm{~s}^{-1}$ is required to achieve perfect absorption in the type 0 structure. Although the type A structure requires lower graphene carrier mobility for perfect absorption than the type 0 structure, such values of graphene carrier mobility are still not easily achievable in processed graphene, and this has limited experimental demonstration of electronically tunable perfect absorption in graphene. Therefore, more advanced designs requiring much lower graphene carrier mobilities are necessary for realizing perfect absorption in graphene.

The Salisbury screen structure in the type 0 and A structures improves coupling radiation into GPRs via a Fabry-Perot interference, and thus induces strongly resonant absorption in GPRs ${ }^{15,16,33}$. While the back reflector has been demonstrated to enhance resonant absorption in GPRs $^{16}$, greater improvements can be achieved by incorporating subwavelength-scale intermediaries $\left(\sim \lambda_{0} / 10, \lambda_{0}\right.$ : free-space wavelength), i.e., noble metal plasmonic antennas ${ }^{37,38}$, which bridge a large wavelength mismatch between free-space photons and graphene plasmons confined in deep-subwavelength-scale GPRs $\left(<\lambda_{0} / 70\right)$, and as a result enhance radiative coupling to graphene nanoresonators. With the noble metal plasmonic light focusing effect, the type B and C metallic slits provide field enhancement factors of 147 and 226 (Supporting Information Part 4), respectively, while a single Salisbury screen increases the field strength by a factor of 4 in theory. The field enhancement can be increased further by widening the metallic strips. However, higher field enhancement does not always guarantee larger absorption in graphene. An optimal field enhancement exists for each structure with a given graphene carrier mobility and 
substrate thickness. Details will be discussed later with the description of the surface admittance model. In the type $\mathrm{B}$ and $\mathrm{C}$ structures, the $\mathrm{SiN}_{x}$ thickness is adjusted to $480 \mathrm{~nm}$, which is smaller in length than quarter wavelength, because the Salisbury screen resonance condition is no longer only determined by the dielectric stack thickness but also by an additional surface inductance from the noble metal plasmonic structures, as discussed below.

Figure $2 \mathrm{a}$ summarizes the calculated absorption in each structure at $1,356 \mathrm{~cm}^{-1}$ as a function of graphene hole mobility at the optimal graphene Fermi levels. The graphene hole mobilities required to achieve perfect absorption are $3,174 \mathrm{~cm}^{2} \mathrm{~V}^{-1} \mathrm{~s}^{-1}, 2,271 \mathrm{~cm}^{2} \mathrm{~V}^{-1} \mathrm{~s}^{-1}, 613 \mathrm{~cm}^{2} \mathrm{~V}^{-}$ ${ }^{1} \mathrm{~s}^{-1}$, and $315 \mathrm{~cm}^{2} \mathrm{~V}^{-1} \mathrm{~s}^{-1}$ for the type $0, \mathrm{~A}, \mathrm{~B}$ and $\mathrm{C}$ structures, respectively. Compared to type 0 and A structures, perfect absorption is achievable at much lower graphene hole mobility in the type B and C structures due to the enhanced field around the GPRs, and these graphene carrier mobilities are accessible with realistic fabrication methods. The type $\mathrm{C}$ slit configuration exhibits a larger field enhancement than the type B slit because the narrower slit in type $\mathrm{C}$ more efficiently confines radiation. As a result, the type $\mathrm{C}$ structure achieves perfect absorption at the lowest graphene hole mobility, and the maximum absorption values with $\mu_{\mathrm{h}}=315 \mathrm{~cm}^{2} \mathrm{~V}^{-1} \mathrm{~s}^{-1}$ for the type 0 , A, and B structures are $50.1 \%, 50.8 \%$, and $91.7 \%$, respectively, as shown in Figure $2 \mathrm{~b}$.

Figure 2c illustrates absorption in each component at the perfect absorption condition for the type $C$ structure, and shows that most absorption (96.0\%) occurs in the GPRs. Although graphene coverage in the type $\mathrm{B}$ and $\mathrm{C}$ structures is less than $10 \%$, their light absorption capacities significantly overwhelm that of an unpatterned graphene sheet, while the coupled noble metal plasmonic structures contribute little to the total absorption (see Supporting Information Part 5 for other structures). Considering the graphene coverage area, the coupled metal structures significantly enhance interband absorption in graphene, and this is another proof of enhanced light-matter interactions in graphene in the type $\mathrm{B}$ and $\mathrm{C}$ structures. Tunable absorption maps of the type $\mathrm{A}, \mathrm{B}$, and $\mathrm{C}$ structures with $\mu_{\mathrm{h}}=315 \mathrm{~cm}^{2} \mathrm{~V}^{-1} \mathrm{~s}^{-1}$ as a function of frequency and graphene Fermi level are shown in Figure 2d-f (see Supporting Information Part 6 for other structures). In the absorption maps, the resonant absorption in graphene blue shifts with higher graphene Fermi level. The large absorption below $1,250 \mathrm{~cm}^{2} \mathrm{~V}^{-1} \mathrm{~s}^{-1}$ originates from phonon modes in $\mathrm{SiO}_{2}$ and $\mathrm{SiN}_{x}$ layers. 
In addition to enhancing field strength, the metallic slits mimic the interaction between adjacent GPRs to create image GPRs inside the metallic stripes shown in Figure 1b,c (Supporting Information Part 4). When GPRs are arrayed periodically without metallic strips, the GPRs interact with adjacent GPRs, and create collective excitations, as shown in Figure 1j. The red regions in Figure $1 \mathrm{j}$ are due to the electric fields induced by the graphene plasmons, and the interaction between the adjacent GPRs corresponds the blue regions. With normally incident light, the electric field in a unit cell is symmetric, and a GPR array is equivalent to a single GPR surrounded by mirror that acts as a perfect electrical conductor. Therefore, we can consider the image GPRs inside the mirror as adjacent virtual GPRs. In the type B and C structures, the metallic stripes operate as mirrors for the GPRs, mimicking the interaction between adjacent GPRs, as shown in Figure 1k,1. In addition to mimicking the interaction between the adjacent GPRs, the metal edges contacting the GPRs in the type $\mathrm{C}$ structure reflect the near-fields induced in the GPRs, and virtually create twice wider GPRs. As a result, the metallic strips effectively create collective modes, such that the GPRs in the type A, B and C structures are equivalent, and accordingly all structures exhibit an equivalent graphene plasmonic resonance condition, affected by the interaction between the adjacent GPRs as well as the GPR width, but the fields are many times stronger in the type $\mathrm{B}$ and $\mathrm{C}$ structures.

Full-wave simulations show large absorption enhancement in the proposed structures, as multi-scale nanophotonic structures composed of a low permittivity substrate and subwavelength noble metal plasmonic antennas significantly improve the radiative coupling to deep subwavelength GPRs. To fully understand the underlying photonic concepts and design the proposed structure, we develop a graphical method based on effective surface admittance, elucidating the physical origin of the perfect absorption and the role of other elements of the proposed multi-scale structure. The electromagnetic admittance is defined as the ratio of the transverse magnetic field to the transverse electric field. Admittance analysis has been widely used in electromagnetic design of multi-layered stacks in response to an incoming wave. The admittance of a finite-thickness layer is usually a complex value, and the real and imaginary part of the admittance correspond to the conductance and susceptance of the layer, respectively. Here, the conductance and the susceptance represent the magnitude and the phase relation of the electromagnetic response, respectively. 
Since the thickness of the graphene plasmonic nanostructures are much thinner than the free-space wavelength, the top layers can be modeled by effective surface admittance $e^{16,17,33,39}$. Figure $3 \mathrm{a}, \mathrm{b}$ show the surface admittance of the type $\mathrm{C}$ structure with $\mu_{\mathrm{h}}=315 \mathrm{~cm}^{2} \mathrm{~V}^{-1} \mathrm{~s}^{-1}$ calculated from full-wave simulations and the surface admittance fitted by a modified susceptibility model. Using the effective surface admittance, we can calculate the absorption by evaluating the interaction between the graphene plasmonic nanostructure and the Salisbury screen. The reflection coefficient of the structure in terms of admittances normalized by air admittance $\left(Y_{0}\right)$ is derived as

$$
r=-\frac{Y_{\mathrm{S}}+Y_{\mathrm{sub}}-Y_{0}}{Y_{\mathrm{S}}+Y_{\mathrm{sub}}+Y_{0}}=-\frac{\tilde{Y}_{\mathrm{S}}+\tilde{Y}_{\text {sub }}-1}{\tilde{Y}_{\mathrm{s}}+\tilde{Y}_{\text {sub }}+1}=-\frac{\tilde{Y}_{\mathrm{L}}-1}{\tilde{Y}_{\mathrm{L}}+1}
$$

where $\tilde{Y}_{\text {sub }}$ is a substrate admittance determined by the substrate geometry (Supporting Information Part 7). Eq. (1) shows perfect absorption is achieved when the surface admittance $\tilde{Y}_{\mathrm{S}}$ is equal to $1-\tilde{Y}_{\text {sub }}$, and the dotted black line in Figure $3 \mathrm{c}$ corresponds to the $1-\tilde{Y}_{\text {sub }}$, which we term a critical line, as a function of the $\operatorname{SiN}_{x}$ thickness $\left(d_{\mathrm{SiN} x}\right)$.

This graphical approach offers an intuitive understanding of the physical origin of perfect absorption in the graphene and the role of other elements of our multi-scale structure. In the surface admittance charts of Figure $3 \mathrm{c}$ for the type $\mathrm{C}$ structure, perfect absorption is achieved at a crossing of the surface admittance line and the critical line, and the maximum surface conductance is strongly dependent on the graphene hole mobility (see Supporting Information Part 7 for other structures). For a critical graphene hole mobility $\left(\mu_{\mathrm{h}, \mathrm{c}}\right)$, the surface admittance and the critical lines form a single critical coupling point at a critical substrate thickness, where perfect absorption occurs. If the graphene hole mobility is lower than the critical graphene hole mobility, the surface admittance line does not cross the critical line, and it corresponds to an under-coupled regime. When $\mu_{\mathrm{h}}>\mu_{\mathrm{h}, \mathrm{c}}$, two critical coupling points exist and deviate from the critical substrate thickness ${ }^{16}$ (see also Supporting Information Part 7). In this regime, the resonance at the critical substrate thickness is over-coupled, which explains why the absorption declines after perfect absorption point in Figure 2a. Further discussion of the surface admittance can be found in Supporting Information Part 8.

Critical coupling can be interpreted as an admittance matching condition. Presenting the load admittance $\tilde{Y}_{\mathrm{L}}$, represented by $\tilde{Y}_{\mathrm{S}}+\tilde{Y}_{\text {sub }}$, the critical coupling condition thus corresponds to matching the load admittance to the free-space admittance. Assuming there is no absorption in 
the dielectric stack and assuming a perfect back reflector, the real part of the substrate admittance becomes zero, and only the imaginary part of the substrate admittance varies, depending on the substrate thickness. Here, the role of the tunable graphene plasmonic nanostructure is to adjust the load admittance so that $\operatorname{Re}\left(\tilde{Y}_{\mathrm{s}}\right)=1$, and the non-zero imaginary part of the $\tilde{Y}_{\mathrm{s}}$ induced by the surface nanostructure is compensated by the substrate admittance, matching the load admittance to free space.

The surface admittance chart of all structures with $\mu_{\mathrm{h}}=315 \mathrm{~cm}^{2} \mathrm{~V}^{-1} \mathrm{~s}^{-1}$ is shown in Figure $3 \mathrm{~d}$, which illustrates the important role of the coupled metallic structures. The type B and C structure exhibits much larger maximum surface conductances, or stronger optical resonances, than the type 0 and $A$ structures due to the enhanced radiative coupling to graphene nanoresonators. We note that the net surface susceptance $\operatorname{Im}\left(\tilde{Y}_{\mathrm{s}, \infty}\right)$ in Figure $3 \mathrm{~b}$ is determined by surface nanostructures without graphene plasmonic resonances. To be specific, the type B and C structures are more inductive (i.e., more negative surface susceptance) than the type 0 and A structures because of the strong noble metal plasmonic resonance induced by the subwavelength metallic slits ${ }^{40,41}$. For the weak scattering interactions with nearly zero net surface susceptance exhibited by the type 0 and A structures, the admittance matching condition is satisfied when the thickness of the substrate meets the quarter wavelength condition of a Salisbury screen. On the other hand, in the type B and C structures, the net surface susceptance is large and negative, indicating radiation coupled by the metallic slits significantly advances the phase of light passing through the slits. Due to this abrupt phase advance in these type B and C structures, admittance matching occurs with thinner substrates compared to weakly scattering Salisbury screens.

A graphical surface admittance analysis also predicts that absorption is dominated by graphene. In Figure 3a,c, the primary source of the surface conductance at maximum absorption is attributed to the graphene plasmonic resonance, while at minimum absorption the surface conductance is very low and no graphene plasmonic resonance is present, indicating the important role of the graphene plasmonic resonance in determining overall absorption.

We can further lower the graphene hole mobility required to achieve perfect absorption by optimizing the geometry of the type C structure (Supporting Information Part 9). The field enhancement inside the subwavelength metallic slits increases for wider metallic stripes because of a larger cross-section, and leads to larger radiative coupling to graphene nanoresonators. As a result, the structure becomes more optically conductive at a given graphene carrier mobility, as 
shown in Figure 4a. Although we can improve the field enhancement by narrowing the metal gap width for higher intergap confinement, reducing the width of the GPRs weakens the oscillator strength, thereby degrading resonant absorption. By tailoring the geometry of the type $\mathrm{C}$ structure, we achieve perfect absorption with $\mu_{\mathrm{h}}=200 \mathrm{~cm}^{2} \mathrm{~V}^{-1} \mathrm{~s}^{-1}$, as shown in Figure $4 \mathrm{~b}$. Other designs could allow for perfect absorption with even lower graphene hole mobility, which indicates that perfect absorption in the graphene plasmonic nanostructures is no longer limited by the low graphene carrier mobility.

To demonstrate electronically tunable perfect absorption in graphene, we measured the absorption $(A=1-R)$ for type A structures fabricated on $\mathrm{SiO}_{2} 150 \mathrm{~nm} / \mathrm{SiN}_{x} 1 \mu \mathrm{m}$, and type $\mathrm{B}$ and C structures fabricated on $\mathrm{SiO}_{2} 150 \mathrm{~nm} / \mathrm{SiN}_{x} 500 \mathrm{~nm}$ in a Fourier transform infrared (FTIR) microscope with a polarizer. The modulation efficiencies in reflection are calculated by $\eta_{R}=1$ $R / R_{\max }$. Here, $R$ corresponds to the gate voltage-dependent reflectance, and $R_{\max }$ is the reflectance when the absorption is minimized at a given graphene Fermi level between the interband absorption and the graphene plasmonic resonance. The graphene Fermi level position is calculated using a capacitor model based on the graphene carrier density obtained from measurements of gate voltage-dependent resistance of the graphene ${ }^{42}$ (Supporting Information Part 10).

The gate voltage-dependent tunable absorption spectra are shown in Figure 5a-c, and the corresponding modulation efficiencies $\left(\eta_{R}\right)$ are shown in Figure 5d-f (Supporting Information 11). Table 1 summarizes the measurement results for the type A, B, and C structures. In type B structures, we also observed the higher-order graphene plasmonic resonance mode ${ }^{10,11}$, which is not easily observable in the type 0 and A structures for low graphene hole mobility (Supporting Information Part 12). Figure $5 \mathrm{~g}$ summarizes the absorption measurements as a function of graphene Fermi level, and corresponding modulation efficiencies are presented in Figure 5h. The measurement results indicate that the subwavelength metallic slits significantly enhance absorption in the GPRs, and both the type B and C structures display nearly perfect absorption. In particular, the type A structure reported here enables higher tunability than our previously measured type 0 structure $^{16}$, which indicates that the low permittivity substrate improves coupling efficiency between free-space photons and graphene plasmons. In addition, the measurement results in Figure $5 \mathrm{~g}$ show an increased baseline absorption in the type B and $\mathrm{C}$ 
structures, primarily due to the interband absorption in graphene enhanced by the plasmonic antenna effect of the metal slits, as predicted by theoretical calculations.

These mid-infrared measurements demonstrate how low permittivity substrates and coupled subwavelength metallic slits play pivotal roles in enhancing the resonant absorption in GPRs. In order to analyze these data further, we set the graphene hole mobilities in our simulations as a fitting parameter, and fit our simulations to the experimental modulation efficiency spectra (Supporting Information Part 13). The fitting results reveal that the type A structure is in the under-coupled regime, and the type B structure is close to the critical coupling condition, which results in nearly perfect absorption in the later. The type $\mathrm{C}$ structure is expected to be in the over-coupled regime (or, the load admittance exceeds air admittance), which explains the lower absorption in the type $\mathrm{C}$ structure than in the type B structure in experimental measurements. Significantly, in order to enhance the resonant absorption in the type C structure, the graphene hole mobility should be decreased rather than increased to achieve the critical coupling. These results indicate that our multi-scale nanophotonic designs have passed a critical threshold, whereby modulation efficiency is no longer limited by intrinsic material properties, and new, less stringent optical designs are likely possible.

We have experimentally demonstrated electronically tunable perfect absorption in graphene. Our graphical design approach enables perfect absorption in graphene even for the low graphene carrier mobility and with less than $10 \%$ of the surface area covered by graphene. In addition, our nanophotonic design for improving radiative coupling to nanostructures, based on combinations of carefully tailored multi-scale resonant elements, constitutes a general approach that can achieve electronically tunable strong light-matter interactions in a broad class of both two-dimensional and thin film materials whose carrier densities and optical properties can be modulated by electrostatic gate control. The fundamental insights found here concerning interaction between metallic nanostructures and graphene have potentially far-reaching applications in graphene-based active infrared optical components, such as modulators, phased arrays, and thermal radiation management structures, as well as similar structures realized using other materials suffering from inherently weak light-matter interactions. 


\begin{abstract}
Methods
Material modeling in simulation

The frequency dependent dielectric functions of $\mathrm{SiO}_{2}$ and $\mathrm{SiN}_{x}$ were obtained from midinfrared spectroscopic ellipsometry, and the dielectric function of $\mathrm{Au}$ was taken from Palik data $^{43}$. The optical surface conductivity of graphene is calculated by random phase approximation $^{44,45}$.
\end{abstract}

\title{
Sample fabrication
}

The proposed structures were fabricated on $1 \mu \mathrm{m}$ of $\mathrm{SiN}_{x}$ membrane (Norcada, $\mathrm{NX10500F}$ ) and $500 \mathrm{~nm}$ of $\mathrm{SiN}_{x}$ membrane (Norcada, NX10500E). The top $150 \mathrm{~nm}$ layer of $\mathrm{SiO}_{2}$ was deposited by e-beam evaporation $(115 \mathrm{~nm})$ and atomic layer deposition $(35 \mathrm{~nm})$. The Ti $3 \mathrm{~nm} / \mathrm{Au} 100 \mathrm{~nm}$ layers were deposited on the back side of the membrane by e-beam evaporation, which performed as a back gate for doping graphene as well as a back reflector for the Salisbury screen. After transferring CVD-grown graphene onto the substrate, the GPRs were patterned by an e-beam lithography system using a PMMA resist. With the patterned PMMA layer serving as a soft etch mask, the GPRs were cut by reactive ion etching. The subwavelength metallic slits were also patterned by e-beam lithography, and the Ti $2 \mathrm{~nm} / \mathrm{Au} 80 \mathrm{~nm}$ layers were deposited by e-beam evaporation. 


\section{FIGURES}

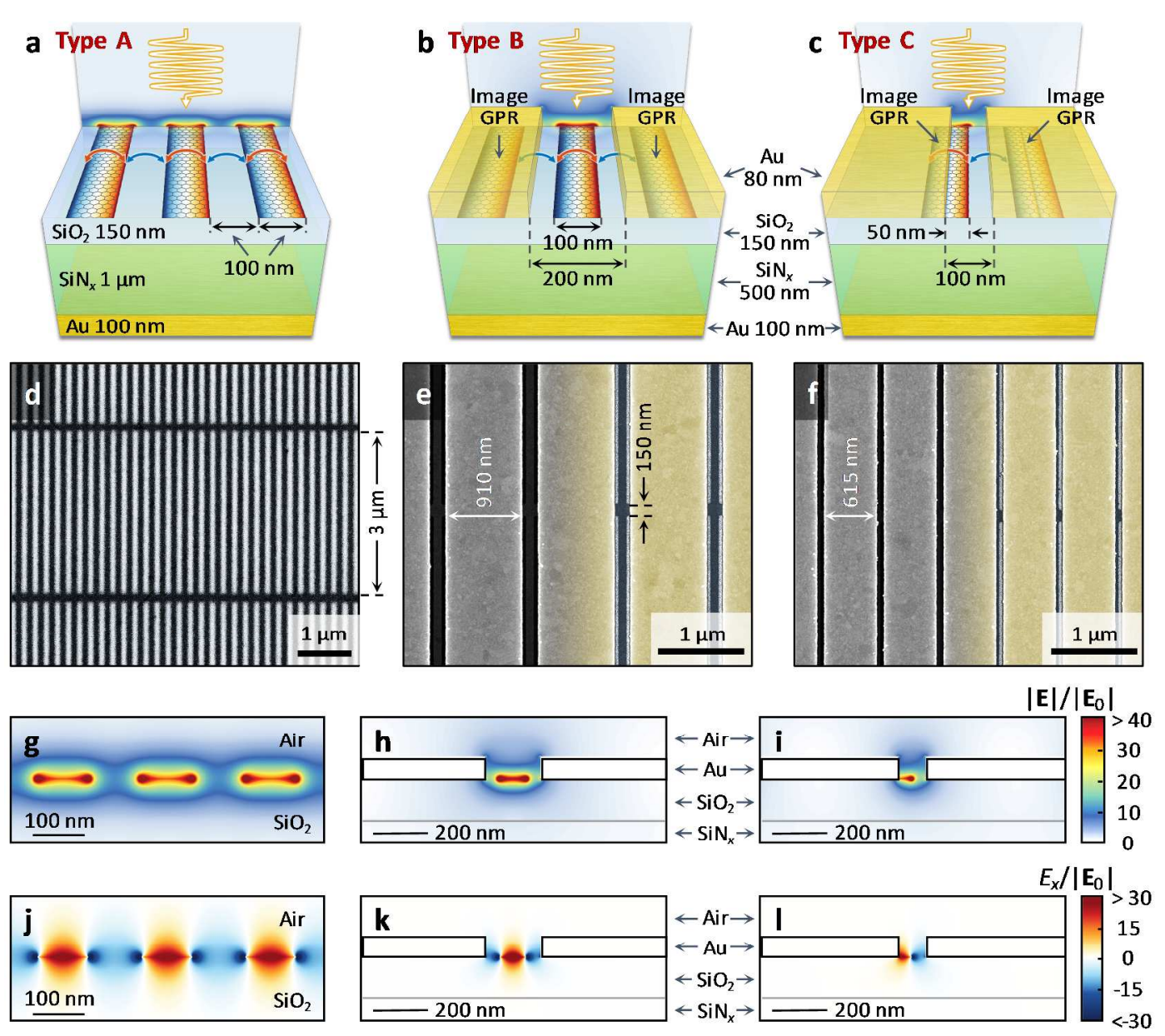

Figure 1. Schematic of (a-c) type A, B, and C structures, respectively, and (d-f) corresponding scanning electron microscope (SEM) images (false color). (g-i) Electric field distributions and (j1) $E_{x}$ distributions in the type $\mathrm{A}, \mathrm{B}$, and $\mathrm{C}$ structures, respectively, with the perfect absorption conditions. Additional field profiles with varying graphene carrier mobilities are presented in Supporting Information Part 2. In (a)-(c), panels at the back side give the out-of-plane electric field distributions, and $E_{z}$ distributions in graphene are overlapped on graphene plasmonic ribbons (GPRs). The image GPRs in (b) and (c) are created by metallic stripes which operate as mirrors creating virtual GPRs corresponding to the GPRs located in the slits. In the SEM images, the dark and bright regions correspond to GPRs and exposed $\mathrm{SiO}_{2}$ areas, respectively. The GPRs have $150 \mathrm{~nm}$ wide bridges to prevent electrical disconnections, and the length of the GPR strip is $3 \mu \mathrm{m}$. In panels (e) and (f), the left side of each image is the original SEM image, whereas 
contrast- and color-adjusted SEM images are overlapped on the right side to enhance visibility of the GPRs.
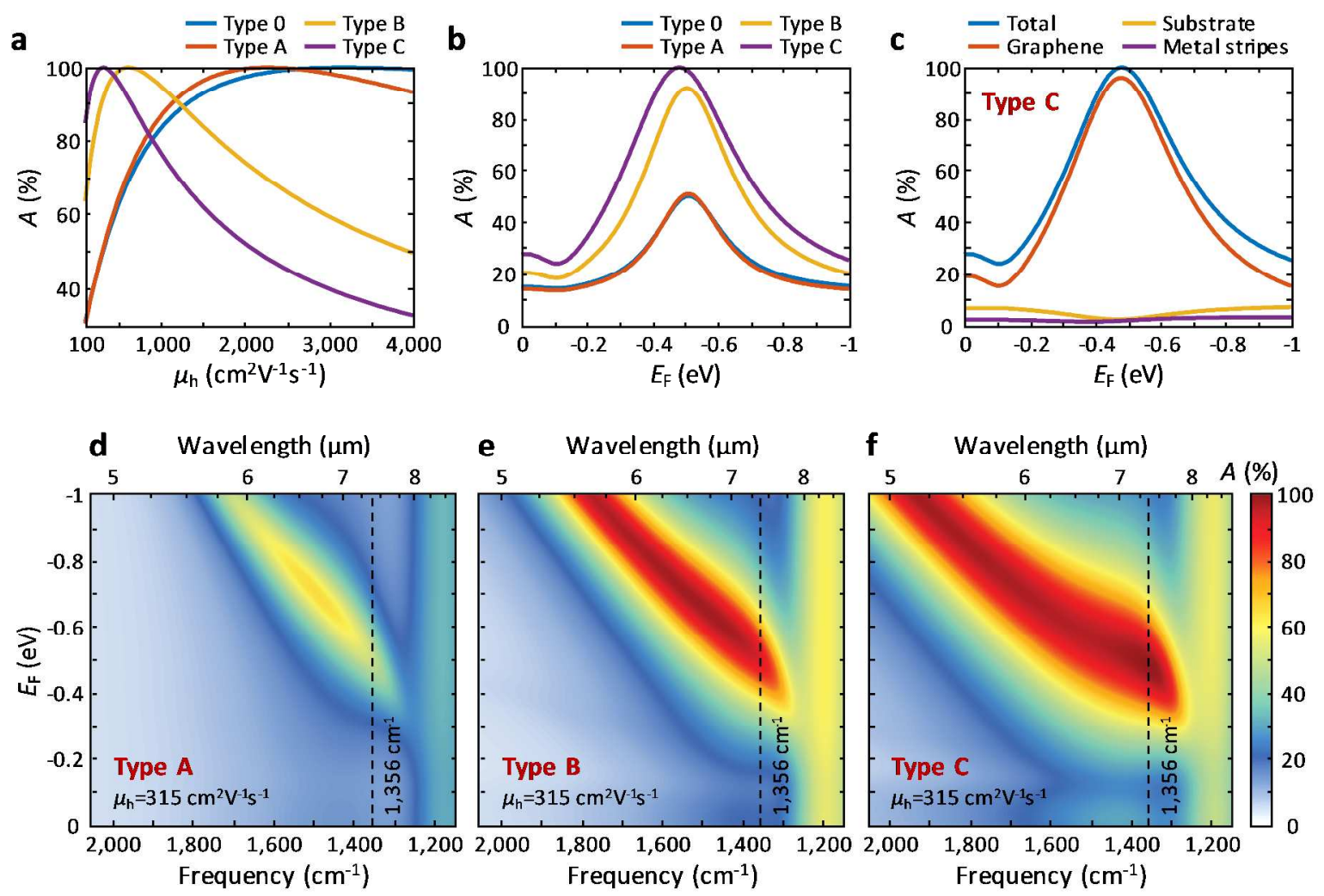

Figure 2. (a) Absorption in each structure at $1,356 \mathrm{~cm}^{-1}$ as a function of graphene hole mobility $\left(\mu_{\mathrm{h}}\right)$. (b) Tunable absorption in each structure as a function of graphene Fermi level $\left(E_{\mathrm{F}}\right)$ for $\mu_{\mathrm{h}}=315 \mathrm{~cm}^{2} \mathrm{~V}^{-1} \mathrm{~s}^{-1}$. (c) Absorption in each component for the type $\mathrm{C}$ structure at $1,356 \mathrm{~cm}^{-1}$ with $\mu_{\mathrm{h}}=315 \mathrm{~cm}^{2} \mathrm{~V}^{-1} \mathrm{~s}^{-1}$ as a function of graphene Fermi level. The absorption in the substrate indicates the combined absorptions in the $\mathrm{SiO}_{2}, \mathrm{SiN}_{x}$, and back reflector layers. (d-f) Absorption maps in the type $\mathrm{A}, \mathrm{B}$, and $\mathrm{C}$ structures, respectively, with $\mu_{\mathrm{h}}=315 \mathrm{~cm}^{2} \mathrm{~V}^{-1} \mathrm{~s}^{-1}$ as a function of frequency and graphene Fermi level $\left(E_{\mathrm{F}}\right)$. 

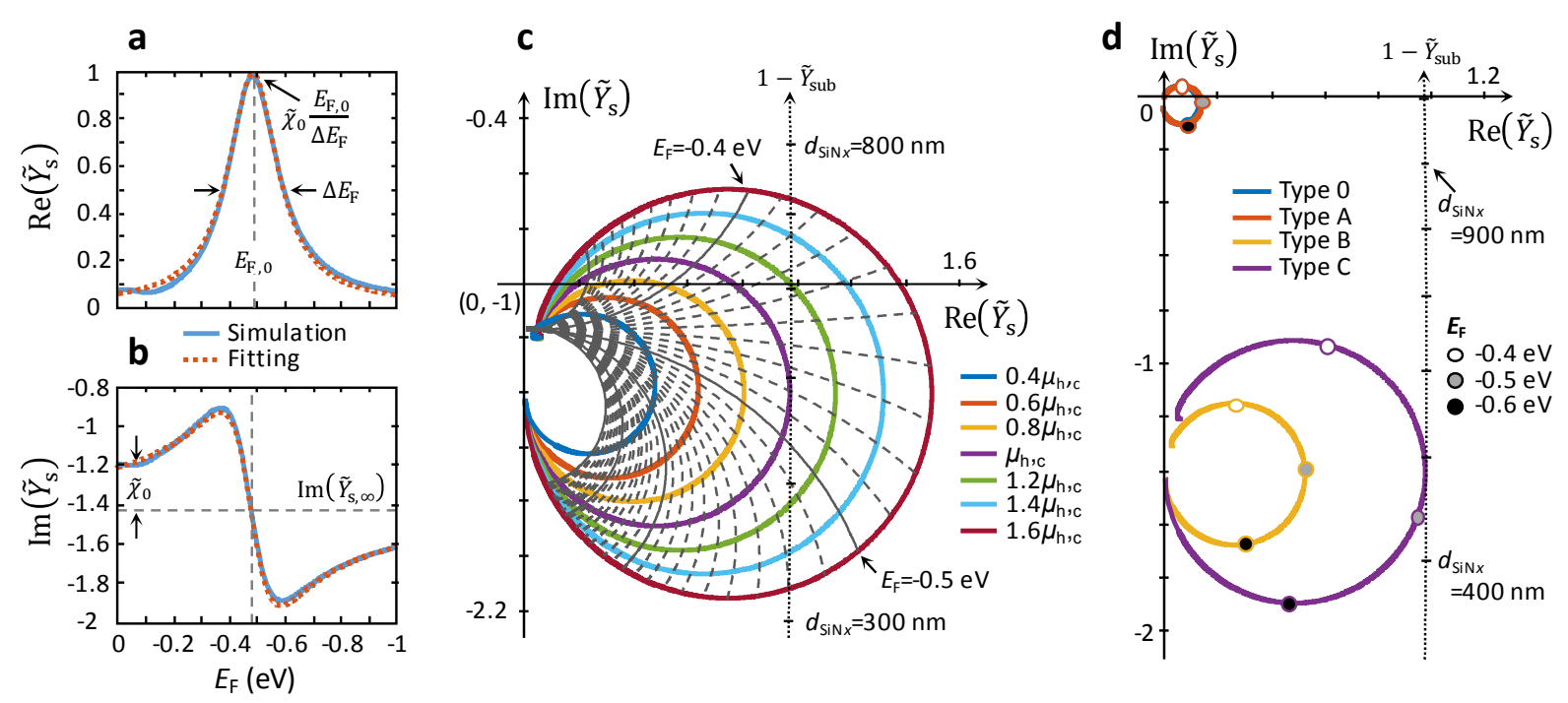

Figure 3. (a) The real part and (b) imaginary part of the surface admittance of the type C structure with the critical graphene hole mobility $\left(\mu_{\mathrm{h}, \mathrm{c}}\right)$. The fitting parameters for the modified susceptibility model in eq S12 of Supporting Information Part 6 are $\tilde{Y}_{\mathrm{s}, \infty}=0.007-1.431$, $\tilde{\chi}_{0}=0.440, E_{\mathrm{F}, 0}=-0.483 \mathrm{eV}$, and $\Delta E_{\mathrm{F}}=0.216 \mathrm{eV}$. (c) Surface admittance chart of the type C structure at $1,356 \mathrm{~cm}^{-1}$ with critical graphene hole mobility $\left(\mu_{\mathrm{h}, \mathrm{c}}\right)$ of $315 \mathrm{~cm}^{2} \mathrm{~V}^{-1} \mathrm{~s}^{-1}$; also shown are circles for various graphene hole mobilities $\left(\mu_{\mathrm{h}}\right)$. The surface admittances are calculated for graphene Fermi levels from $0 \mathrm{eV}$ to $-20 \mathrm{eV}$, and the equi- $E_{\mathrm{F}}$ lines from $-0.3 \mathrm{eV}$ to $-0.8 \mathrm{eV}$ with $0.01 \mathrm{eV}$ steps (dotted grey lines) and $0.1 \mathrm{eV}$ steps (solid grey lines). (d) Surface admittance chart of all structures at $1,356 \mathrm{~cm}^{-1}$ with $\mu_{\mathrm{h}}=315 \mathrm{~cm}^{2} \mathrm{~V}^{-1} \mathrm{~s}^{-1}$. The white, gray, and black dots in each surface admittance correspond to $E_{\mathrm{F}}=-0.4 \mathrm{eV},-0.5 \mathrm{eV}$, and $-0.6 \mathrm{eV}$, respectively. 

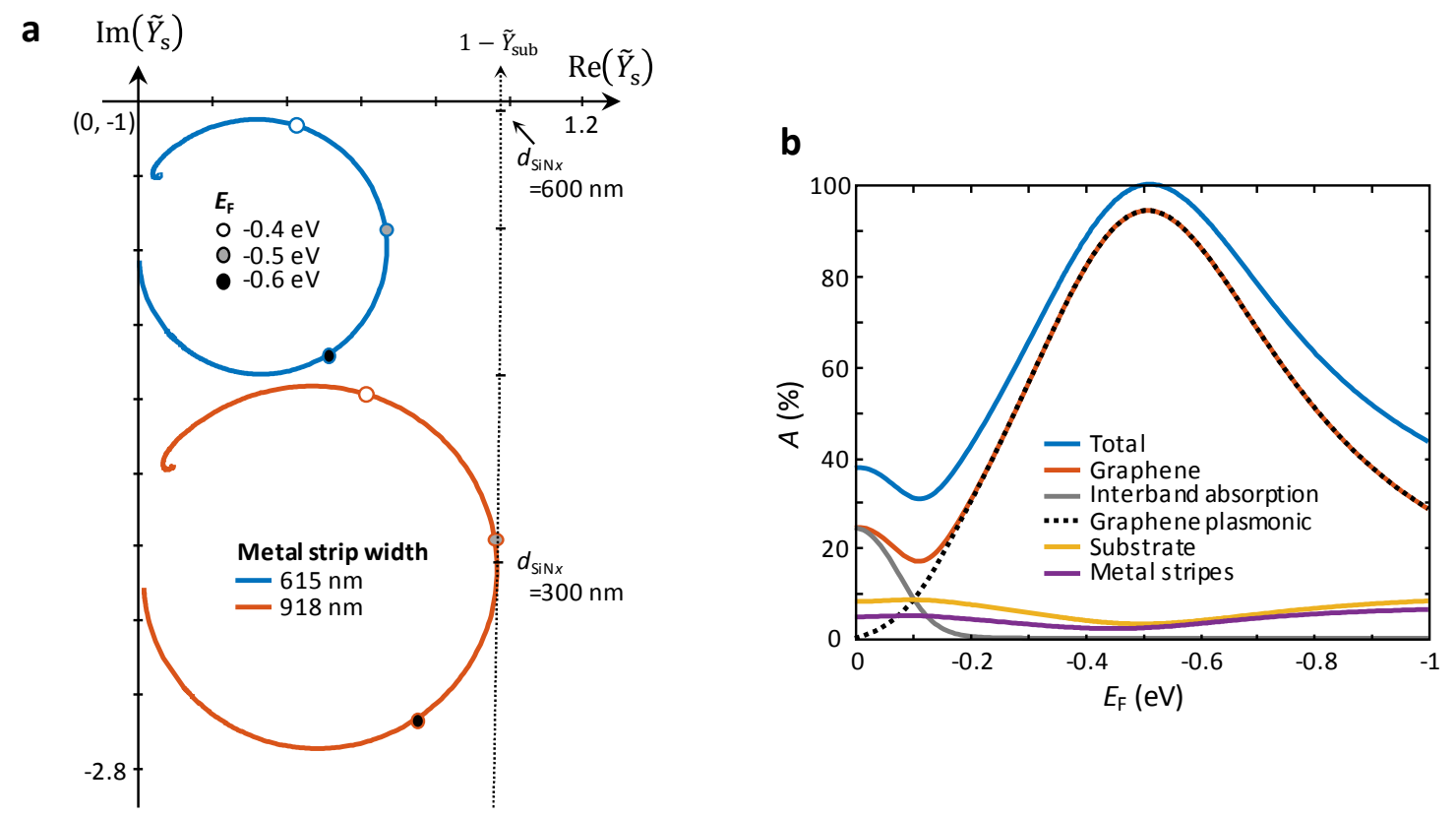

Figure 4. (a) Surface admittance chart of the type $\mathrm{C}$ structures on the $\mathrm{SiO}_{2} 150 \mathrm{~nm} / \mathrm{SiN}_{x}$ substrate having different metal strip widths (blue: $615 \mathrm{~nm}$, red: $918 \mathrm{~nm}$ ) with graphene hole mobility of $200 \mathrm{~cm}^{2} \mathrm{~V}^{-1} \mathrm{~s}^{-1}$ at $1,356 \mathrm{~cm}^{-1}$. The graphene ribbon width, the metallic slit width and the metal thickness are $50 \mathrm{~nm}, 100 \mathrm{~nm}$, and $80 \mathrm{~nm}$, respectively, for both structures. The white, gray, and black dots in each surface admittance correspond to $E_{\mathrm{F}}=-0.4 \mathrm{eV},-0.5 \mathrm{eV}$, and $-0.6 \mathrm{eV}$, respectively. (b) Tunable absorption at $1,356 \mathrm{~cm}^{-1}$ in the optimized type $\mathrm{C}$ structure on a $\mathrm{SiO}_{2}$ $150 \mathrm{~nm} / \mathrm{SiN}_{x} 295 \mathrm{~nm} / \mathrm{Au}$ substrate with graphene hole mobility of $200 \mathrm{~cm}^{2} \mathrm{~V}^{-1} \mathrm{~s}^{-1}$ as a function of graphene Fermi level $\left(E_{\mathrm{F}}\right)$. Perfect absorption is observed at $E_{\mathrm{F}}=-0.514 \mathrm{eV}$. The absorption in the substrate indicates the combined absorptions in the $\mathrm{SiO}_{2}, \mathrm{SiN}_{x}$, and back reflector layers. The "Graphene", the "Interband absorption", and the "Graphene plasmonic" in panels denote total absorption in graphene, absorption by interband transition in graphene, and absorption by plasmonic resonance in graphene, respectively. Maximum absorption in graphene at $E_{\mathrm{F}}=-0.514$ $\mathrm{eV}$ is $94.3 \%$, and maximum absorption by the interband transition in graphene is $24.4 \%$. In this structure, the graphene covers $4.91 \%$ of surface area. 

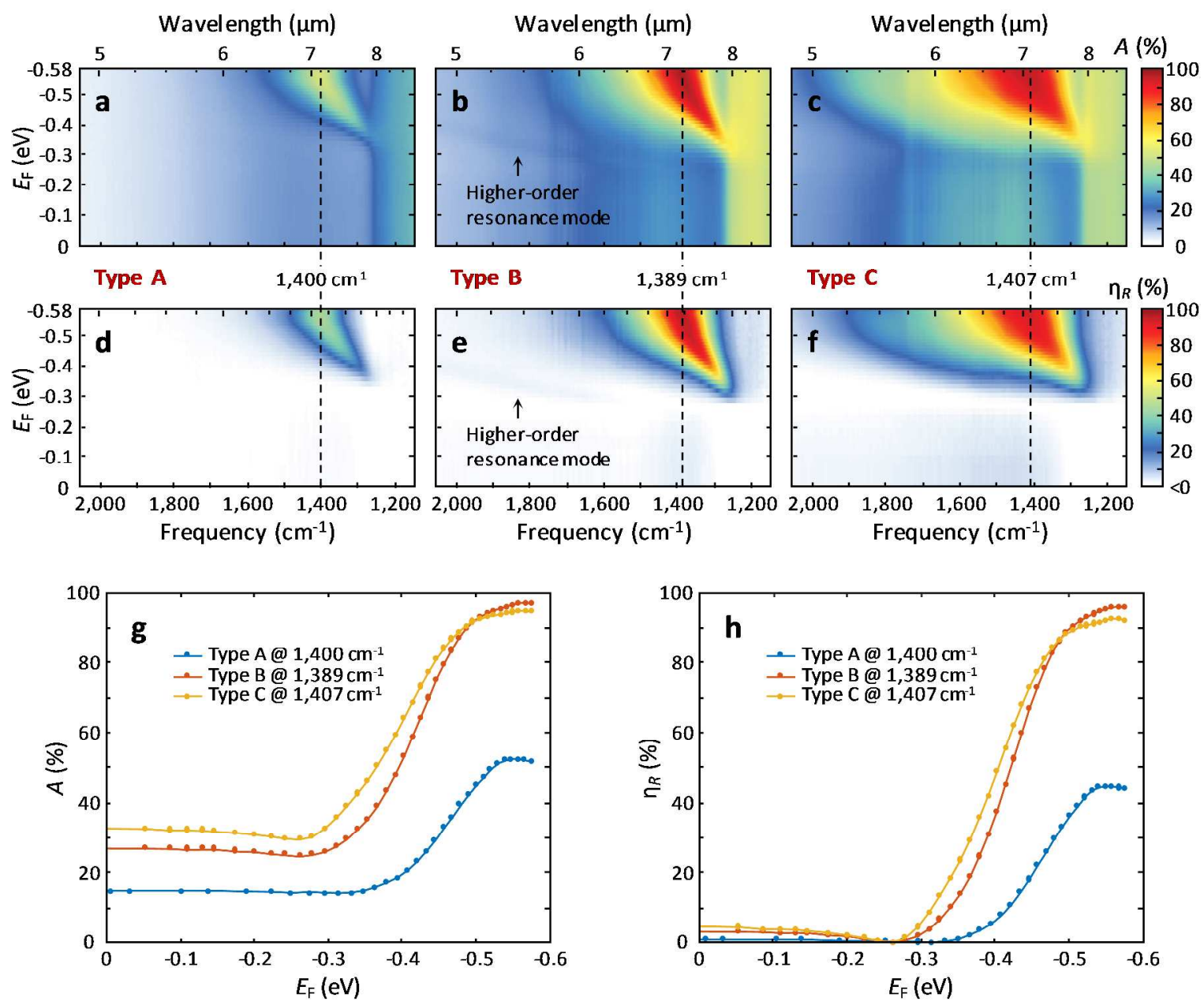

Figure 5. (a-c) Gate voltage-dependent tunable resonant absorption spectra in the type A, B, and $\mathrm{C}$ structures, respectively, and (d-f) corresponding modulation efficiencies $\left(\eta_{R}\right)$. (g) Absorption and (h) modulation efficiency as a function of graphene Fermi level $\left(E_{\mathrm{F}}\right)$ at the frequency for maximum absorption in each structure. 


\section{TABLES}

\begin{tabular}{|c|c|c|c|}
\hline \multicolumn{2}{|l|}{ Table 1. Summary of measurement results } \\
\hline & Type A & Type B & Type C \\
\hline Frequency & $1,400 \mathrm{~cm}^{-1}$ & $1,389 \mathrm{~cm}^{-1}$ & $1,407 \mathrm{~cm}^{-1}$ \\
\hline Maximum absorption $/ \boldsymbol{E}_{\mathbf{F}}$ & $52.4 \% /-0.550 \mathrm{eV}$ & $96.9 \% /-0.568 \mathrm{eV}$ & $94.8 \% /-0.560 \mathrm{eV}$ \\
\hline Minimum absorption $/ \boldsymbol{E}_{\mathbf{F}}$ & $14.0 \% /-0.316 \mathrm{eV}$ & $24.8 \% /-0.262 \mathrm{eV}$ & $29.6 \% /-0.262 \mathrm{eV}$ \\
\hline Modulation efficiency $\left(\boldsymbol{\eta}_{\boldsymbol{R}}\right)$ & $44.6 \%$ & $95.9 \%$ & $92.6 \%$ \\
\hline
\end{tabular}




\section{ASSOCIATED CONTENT}

\section{Supporting Information}

Additional explanation for multi-scale nanophotonic design and surface admittance, additional simulation results, supplementary experimental results (PDF)

\section{AUTHOR INFORMATION}

\section{Corresponding Author}

*E-mail: haa@caltech.edu

\section{Author Contributions}

S.K. and H.A.A. conceived the ideas. S.K., M.S.J., and K.W.M. performed the simulations and formulated the analytic model. S.K. fabricated the sample. S.K., V.W.B., K.W.M., and L.K. performed measurements and data analysis. All authors co-wrote the paper, and H.A.A. supervised the project. ${ }^{+}$S.K., M.S.J., and V.W.B. contributed equally.

\section{Notes}

The authors declare no competing financial interest.

\section{ACKNOWLEDGMENT}

This work was supported by US Department of Energy (DOE) Office of Science grant DEFG02-07ER46405 (S.K., K.W.M., L.K., and H.A.A.), by the Multidisciplinary University Research Initiative Grant, Air Force Office of Scientific Research MURI, Grant No. FA9550-121-0488 (V.W.B.), by Creative Materials Discovery Program through the National Research Foundation of Korea (NRF) funded by the Ministry of Science and ICT (NRF2016M3D1A1900038, M.S.J.), and by Basic Science Research Program through NRF funded by the Ministry of Education (NRF-2017R1D1A1B03034762, M.S.J.). S.K. acknowledges support by a Samsung Scholarship. The authors thank G. Rossman for assistance with the FTIR microscope and W.-H. Lin for assistance with fabrication.

\section{ABBREVIATIONS \\ GPR, graphene plasmonic ribbon.}




\section{REFERENCES}

(1) Fei, Z.; Rodin, A. S.; Andreev, G. O.; Bao, W.; McLeod, A. S.; Wagner, M.; Zhang, L. M.; Zhao, Z.; Thiemens, M.; Dominguez, G.; Fogler, M. M.; Castro Neto, A. H.; Lau, C. N.; Keilmann, F.; Basov, D. N. Nature 2012, 487, 82-85.

(2) García de Abajo, F. J. ACS Photonics 2014, 1, 135-152.

(3) Constant, T. J.; Hornett, S. M.; Chang, D. E.; Hendry, E. Nat. Phys. 2016, 12, 124-127.

(4) Chen, J. N.; Badioli, M.; Alonso-Gonzalez, P.; Thongrattanasiri, S.; Huth, F.; Osmond, J.; Spasenovic, M.; Centeno, A.; Pesquera, A.; Godignon, P.; Elorza, A. Z.; Camara, N.; García de Abajo, F. J.; Hillenbrand, R.; Koppens, F. H. L. Nature 2012, 487, 77-81.

(5) Alonso-Gonzalez, P.; Nikitin, A. Y.; Golmar, F.; Centeno, A.; Pesquera, A.; Velez, S.; Chen, J.; Navickaite, G.; Koppens, F.; Zurutuza, A.; Casanova, F.; Hueso, L. E.; Hillenbrand, R. Science 2014, 344, 1369-1373.

(6) Koppens, F. H. L.; Chang, D. E.; García de Abajo, F. J. Nano Lett. 2011, 11, 3370-3377.

(7) Ju, L.; Geng, B. S.; Horng, J.; Girit, C.; Martin, M.; Hao, Z.; Bechtel, H. A.; Liang, X. G.; Zettl, A.; Shen, Y. R.; Wang, F. Nat. Nanotechnol. 2011, 6, 630-634.

(8) Fang, Z. Y.; Thongrattanasiri, S.; Schlather, A.; Liu, Z.; Ma, L. L.; Wang, Y. M.; Ajayan, P. M.; Nordlander, P.; Halas, N. J.; García de Abajo, F. J. ACS Nano 2013, 7, 2388-2395.

(9) Fang, Z. Y.; Wang, Y. M.; Schather, A. E.; Liu, Z.; Ajayan, P. M.; García de Abajo, F. J.; Nordlander, P.; Zhu, X.; Halas, N. J. Nano Lett. 2014, 14, 299-304.

(10) Yan, H. G.; Li, Z. Q.; Li, X. S.; Zhu, W. J.; Avouris, P.; Xia, F. N. Nano Lett. 2012, 12, 3766-3771.

(11) Yan, H. G.; Low, T.; Zhu, W. J.; Wu, Y. Q.; Freitag, M.; Li, X. S.; Guinea, F.; Avouris, P.; Xia, F. N. Nat. Photonics 2013, 7 , 394-399.

(12) Brar, V. W.; Jang, M. S.; Sherrott, M.; Lopez, J. J.; Atwater, H. A. Nano Lett. 2013, 13, 2541-2547.

(13) Brar, V. W.; Jang, M. S.; Sherrott, M.; Kim, S.; Lopez, J. J.; Kim, L. B.; Choi, M.; Atwater, H. Nano Lett. 2014, 14, 3876-3880.

(14) Brar, V. W.; Sherrott, M. C.; Jang, M. S.; Kim, S.; Kim, L.; Choi, M.; Sweatlock, L. A.; Atwater, H. A. Nat. Commun. 2015, 6, 7032.

(15) Thongrattanasiri, S.; Koppens, F. H. L.; García de Abajo, F. J. Phys. Rev. Lett. 2012, 108, 047401 .

(16) Jang, M. S.; Brar, V. W.; Sherrott, M. C.; Lopez, J. J.; Kim, L.; Kim, S.; Choi, M.; Atwater, H. A. Phys. Rev. B 2014, 90, 165409. 
(17) Yao, Y.; Shankar, R.; Kats, M. A.; Song, Y.; Kong, J.; Loncar, M.; Capasso, F. Nano Lett. 2014, 14, 6526-6532.

(18) Jadidi, M. M.; Sushkov, A. B.; Myers-Ward, R. L.; Boyd, A. K.; Daniels, K. M.; Gaskill, D. K.; Fuhrer, M. S.; Drew, H. D.; Murphy, T. E. Nano Lett. 2015, 15, 7099-7104.

(19) Liu, P. Q.; Luxmoore, I. J.; Mikhailov, S. A.; Savostianova, N. A.; Valmorra, F.; Faist, J.; Nash, G. R. Nat. Commun. 2015, 6, 8969.

(20) Shi, S. F.; Zeng, B.; Han, H. L.; Hong, X.; Tsai, H. Z.; Jung, H. S.; Zettl, A.; Crommie, M. F.; Wang, F. Nano Lett. 2015, 15, 372-377.

(21) Kim, S.; Jang, M. S.; Brar, V. W.; Tolstova, Y.; Mauser, K. W.; Atwater, H. A. Nat. Commun. 2016, 7, 12323.

(22) Yan, H. G.; Li, X. S.; Chandra, B.; Tulevski, G.; Wu, Y. Q.; Freitag, M.; Zhu, W. J.; Avouris, P.; Xia, F. N. Nat. Nanotechnol. 2012, 7, 330-334.

(23) Pirkle, A.; Chan, J.; Venugopal, A.; Hinojos, D.; Magnuson, C. W.; McDonnell, S.; Colombo, L.; Vogel, E. M.; Ruoff, R. S.; Wallace, R. M. Appl. Phys. Lett. 2011, 99, 122108.

(24) Chan, J.; Venugopal, A.; Pirkle, A.; McDonnell, S.; Hinojos, D.; Magnuson, C. W.; Ruoff, R. S.; Colombo, L.; Wallace, R. M.; Vogel, E. M. ACS Nano 2012, 6, 3224-3229.

(25) Novoselov, K. S.; Geim, A. K.; Morozov, S. V.; Jiang, D.; Katsnelson, M. I.; Grigorieva, I. V.; Dubonos, S. V.; Firsov, A. A. Nature 2005, 438, 197-200.

(26) Wang, L.; Meric, I.; Huang, P. Y.; Gao, Q.; Gao, Y.; Tran, H.; Taniguchi, T.; Watanabe, K.; Campos, L. M.; Muller, D. A.; Guo, J.; Kim, P.; Hone, J.; Shepard, K. L.; Dean, C. R. Science 2013, 342, 614-617.

(27) Fang, T.; Konar, A.; Xing, H.; Jena, D. Phys. Rev. B 2008, 78, 205403.

(28) Ouyang, Y. J.; Sanvito, S.; Guo, J. Surf. Sci. 2011, 605, 1643-1648.

(29) Venugopal, A.; Chan, J.; Li, X. S.; Magnuson, C. W.; Kirk, W. P.; Colombo, L.; Ruoff, R. S.; Vogel, E. M. J. Appl. Phys. 2011, 109, 104511.

(30) Fei, Z.; Goldflam, M. D.; Wu, J. S.; Dai, S.; Wagner, M.; McLeod, A. S.; Liu, M. K.; Post, K. W.; Zhu, S.; Janssen, G. C. A. M.; Fogler, M. M.; Basov, D. N. Nano Lett. 2015, 15, 82718276.

(31) Zhu, W. J.; Perebeinos, V.; Freitag, M.; Avouris, P. Phys. Rev. B 2009, 80, 235402.

(32) Wu, C. H.; Neuner, B.; Shvets, G.; John, J.; Milder, A.; Zollars, B.; Savoy, S. Phys. Rev. B 2011, 84, 075102.

(33) Fante, R. L.; McCormack, M. T. IEEE Trans. Antennas Propag. 1988, 36, 1443-1454. 
(34) Jablan, M.; Buljan, H.; Soljacic, M. Phys. Rev. B 2009, 80, 245435.

(35) Gan, C. H.; Chu, H. S.; Li, E. P. Phys. Rev. B 2012, 85, 125431.

(36) Hwang, E. H.; Das Sarma, S. Phys. Rev. B 2007, 75, 205418.

(37) Volpe, G.; Cherukulappurath, S.; Parramon, R. J.; Molina-Terriza, G.; Quidant, R. Nano Lett. 2009, 9, 3608-3611.

(38) Novotny, L.; Van Hulst, N. Nat. Photonics 2011, 5, 83-90.

(39) Andryieuski, A.; Lavrinenko, A. V. Opt. Express 2013, 21, 9144-9155.

(40) Engheta, N.; Salandrino, A.; Alu, A. Phys. Rev. Lett. 2005, 95, 095504.

(41) Georgiou, G.; Tserkezis, C.; Schaafsma, M. C.; Aizpurua, J.; Rivas, J. G. Phys. Rev. B 2015, 91, 125443.

(42) Das, A.; Pisana, S.; Chakraborty, B.; Piscanec, S.; Saha, S. K.; Waghmare, U. V.; Novoselov, K. S.; Krishnamurthy, H. R.; Geim, A. K.; Ferrari, A. C.; Sood, A. K. Nat. Nanotechnol. 2008, 3, 210-215.

(43) Palik, E. D.; Ghosh, G., Handbook of optical constants of solids. Academic Press: San Diego, 1998.

(44) Falkovsky, L. A. J. Phys. Conf. Ser. 2008, 129, 012004.

(45) Falkovsky, L. A.; Varlamov, A. A. Eur. Phys. J. B 2007, 56, 281-284. 

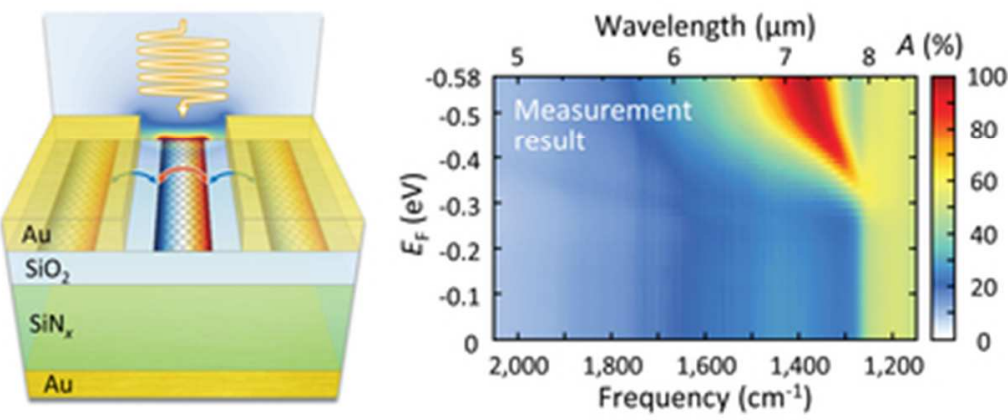

\author{
TOC image \\ $34 \times 14 \mathrm{~mm}(300 \times 300$ DPI $)$
}




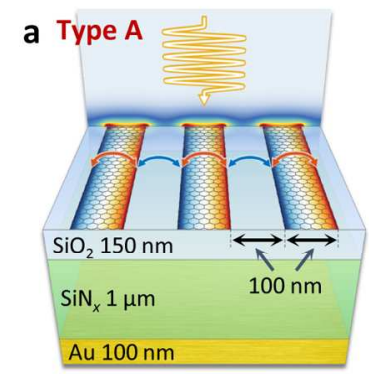

\section{b Type B}
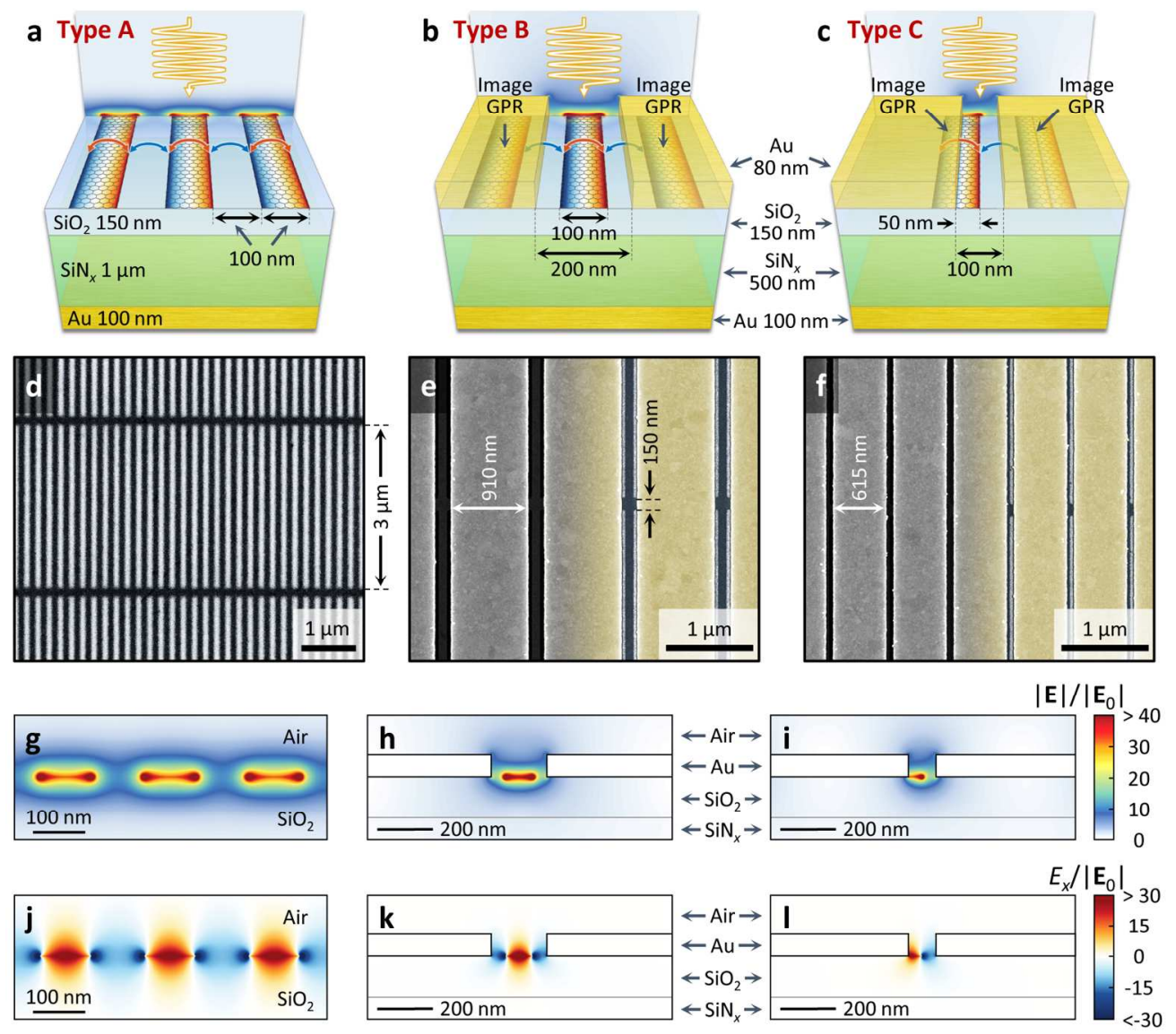

Figure 1

$150 \times 134 \mathrm{~mm}(300 \times 300$ DPI $)$ 

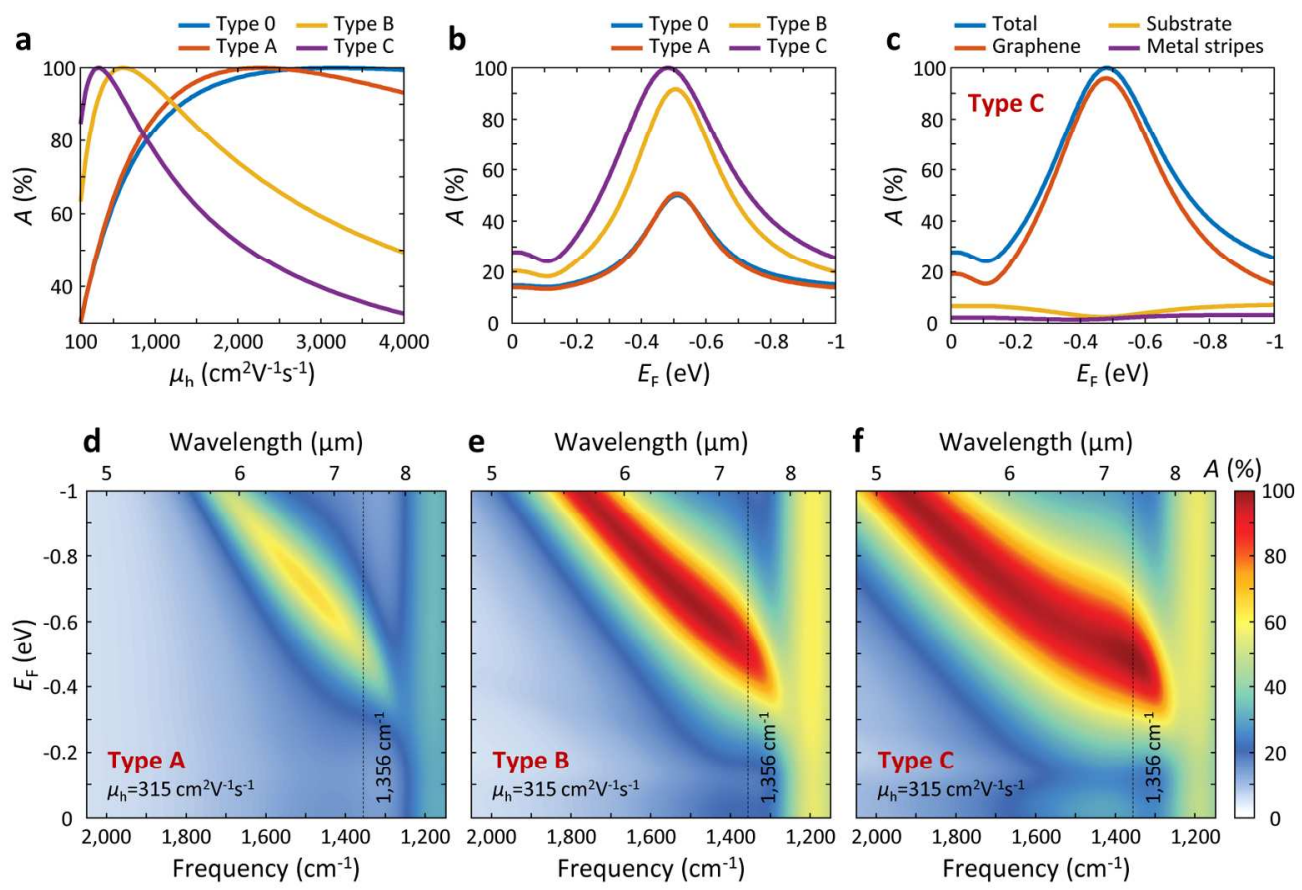

Figure 2

$166 \times 113 \mathrm{~mm}(300 \times 300$ DPI $)$ 

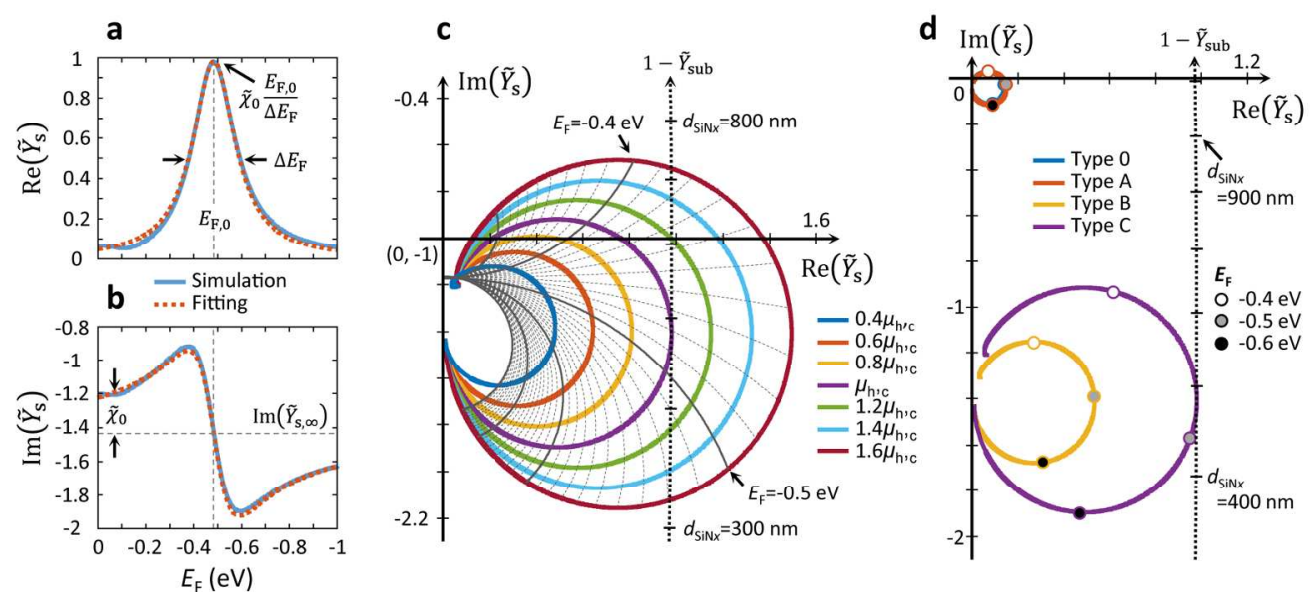

Figure 3

$167 \times 77 \mathrm{~mm}(300 \times 300 \mathrm{DPI})$ 

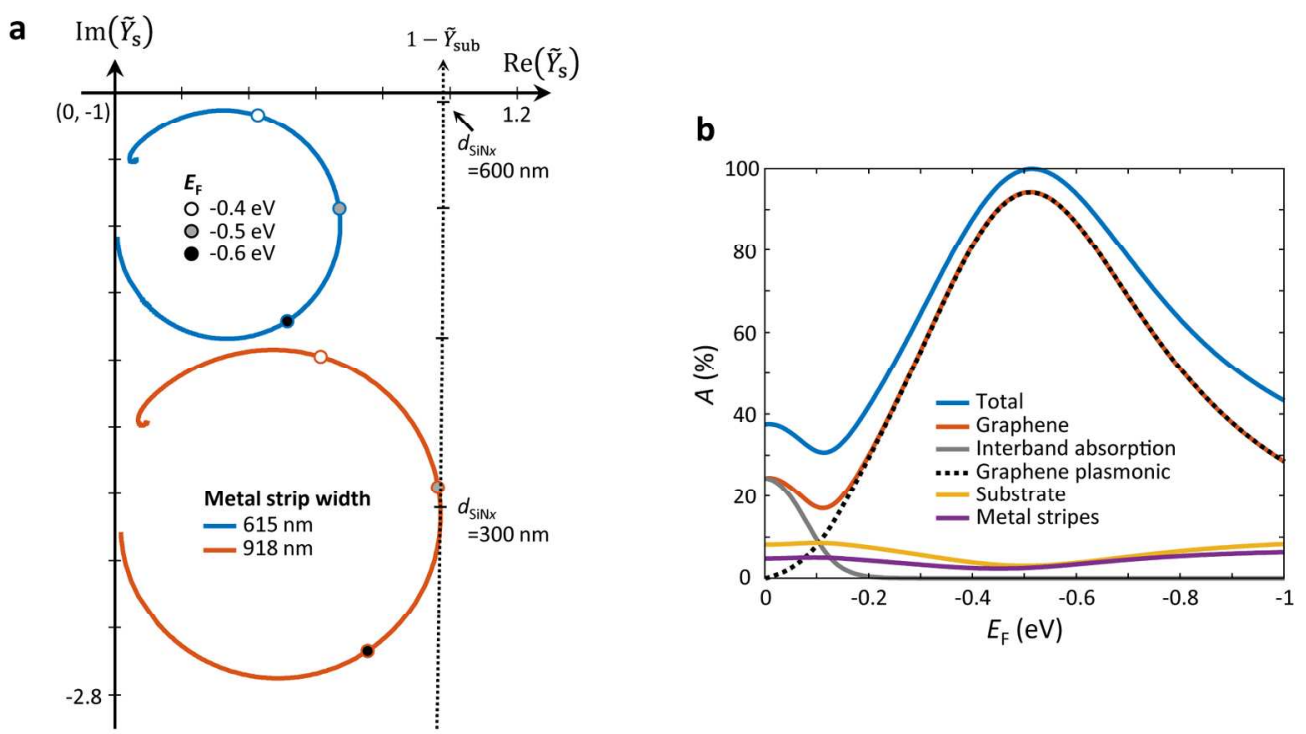

Figure 4

$158 \times 90 \mathrm{~mm}(300 \times 300 \mathrm{DPI})$ 

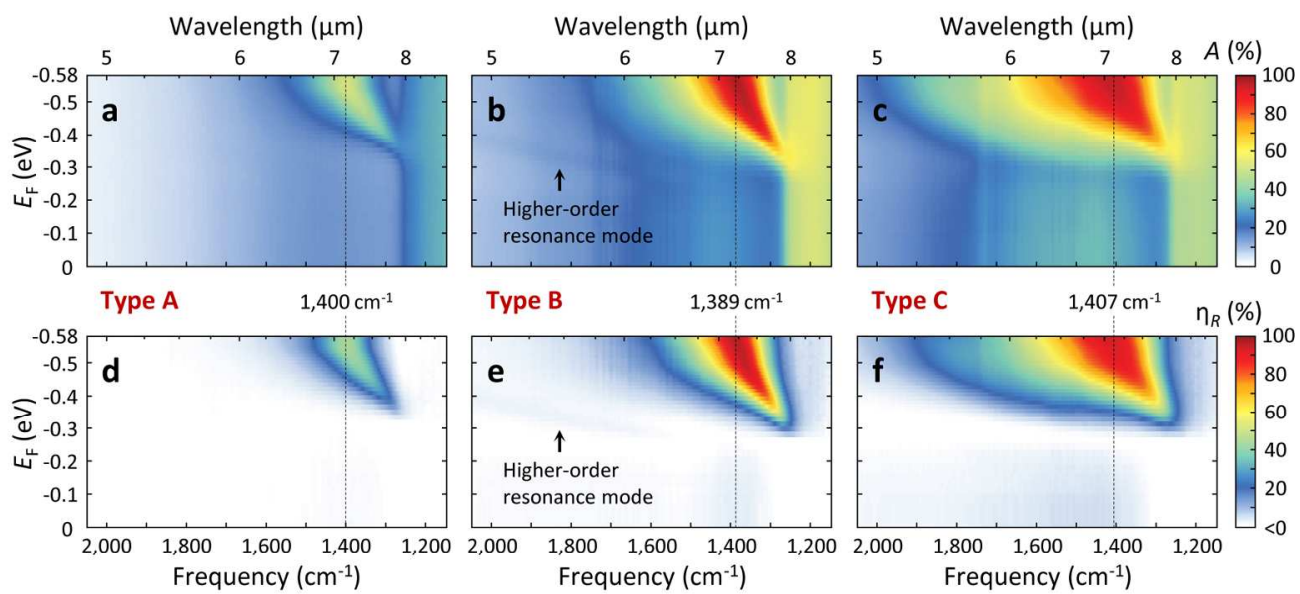

Type B $\quad 1,389 \mathrm{~cm}^{-1}$
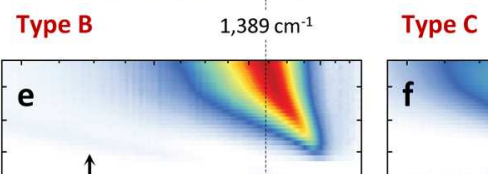

Higher-order

Higher-order
resonance mode
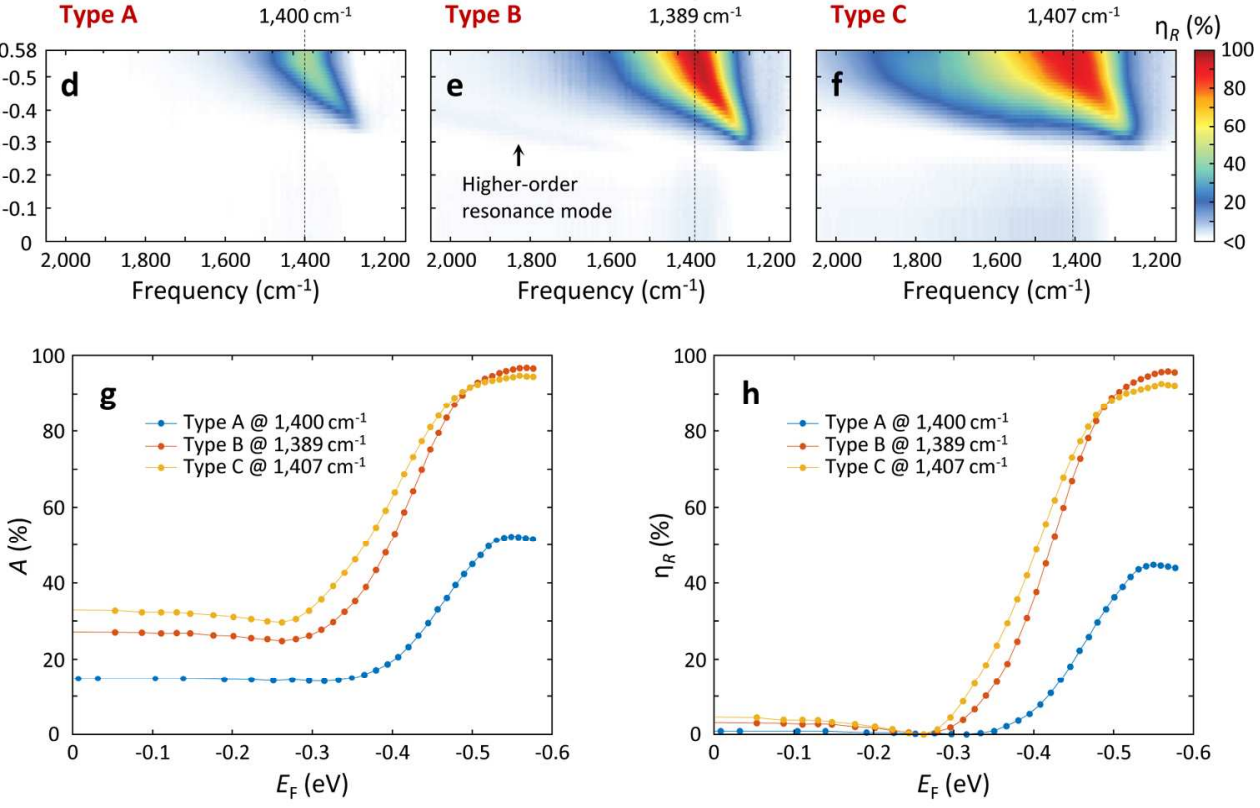

Figure 5

$166 \times 139 \mathrm{~mm}(300 \times 300 \mathrm{DPI})$ 